\title{
Clinical efficacy of administering oxaliplatin combined with S-1 in the treatment of advanced triple-negative breast cancer
}

\author{
JUN LIU, YANG XIAO, WEI WEI, JIAN-XIONG GUO, YANG-CHEN LIU, XIAO-HONG HUANG, \\ RONG-XIA ZHANG, YI-JIA WU and JUAN ZHOU \\ Department of Oncology, Taixing People's Hospital, Medical School of Yangzhou University, \\ Taixing, Jiangsu 225400, P.R. China
}

Received August 11, 2014; Accepted May 1, 2015

DOI: $10.3892 /$ etm.2015.2489

\begin{abstract}
Triple-negative breast cancer (TNBC) is not amenable to current targeted therapies and carries a poor prognosis; however, a specific systemic regimen cannot yet be recommended. The optimal duration of oxaliplatin (OXA) and S-1 combinatorial chemotherapy in patients with advanced breast cancer is not currently known and is likely to be patient-specific based on efficacy and toxicity. In the present study, 52 patients with advanced TNBC received OXA and S-1 chemotherapy. The efficacy and toxicity were observed. The results showed that the median number of regimens was 4 (range 2-6). The therapeutic efficacy was evaluated in all patients. The complete response, partial response, overall response and disease control rates were 3.8, 30.8, 34.6 and $69.2 \%$, respectively. Four patients were lost to follow-up, and the median follow-up time was 13.7 months. The median progression-free survival time was 6.7 months [95\% confidence interval (CI), 4.5-9.0] and the median overall survival (OS) time was 13.3 months (95\% CI, 9.1-17.5). From the subgroup analysis, it was found that the median OS time of patients with stage IV disease and $\geq 2$ metastases was significantly shorter than that of patients with stage IIIC disease and only 1 metastasis [11.3 vs. 22.7 months, $\mathrm{P}=0.010$ (stage IV vs. stage IIC); 11.3 vs. 15.7 months, $\mathrm{P}=0.048$ ( $\geq 2$ vs. 1 metastasis)]. The main grade $3 / 4$ toxic effects were neutropenia (11.5\%), nausea (7.7\%) and nerve toxicity (3.8\%). The other toxic effects were mainly of grades 1-2 and included diarrhea, liver dysfunction, stomatitis, anemia and hand-foot syndrome. In conclusion, OXA combined with $\mathrm{S}-1$ is an effective and tolerable regimen for the treatment of patients with advanced TNBC.
\end{abstract}

Correspondence to: Dr Yang Xiao, Department of Oncology, Taixing People's Hospital, Medical School of Yangzhou University, 1 Chang Zheng Road, Taixing, Jiangsu 225400, P.R. China

E-mail: xiaoyang201407@163.com

Key words: oxaliplatin, S-1, advanced triple-negative breast cancer, chemotherapy

\section{Introduction}

Triple-negative breast cancer (TNBC), defined by a lack of estrogen receptor (ER), progesterone receptor (PR) and human epidermal growth factor receptor 2 (HER-2) expression, is a clinically distinct subgroup accounting for $12-24 \%$ of breast cancer cases $(1,2)$. The majority of these tumors have an inherent aggressiveness, an increased likelihood of distant recurrence, mortality within 5 years of the diagnosis and a poor prognosis (3). Since TNBC is not sensitive to hormone therapy and there is a lack of HER2-targeted therapies, chemotherapy is the only systemic treatment of choice; however, no formal treatment guidelines exist regarding the specific most appropriate systemic regimen for TNBC. Chemotherapy drugs can be administered as a conventional monotherapy or in combination and constitute an important therapeutic tool. The efficacy of anthracycline- and taxane-based neoadjuvant therapies has been confirmed for the treatment of TNBC (4); however, previous research has shown that treatment with anthracycline carries a poor prognosis (5). The improvement of overall survival (OS) rates among patients with advanced TNBC has therefore become a hotspot in breast cancer research.

In a study by Liu et al (6), it was reported that TNBC exhibited increased sensitivity to platinum-based drugs. Oxaliplatin (OXA), as a third-generation platinum-based drug, has no complete cross-resistance with cisplatin or carboplatin and shows synergy with 5-fluorouracil (5-FU) (7), although OXA is effective even in those cases showing resistance to 5-FU. $5-\mathrm{FU}$ is one of most commonly used drugs for breast cancer. $\mathrm{S}-1$ is an oral 5-FU anti-cancer drug with superior efficacy to tegafur (FT) and 5-FU (8). As a monotherapy, S-1 has been associated with a high rate of efficacy, mild adverse effects and good tolerance (9). The aim of this retrospective study, therefore, was to evaluate the effect of an OXA- and S-1-based chemotherapy regimen in the treatment of advanced TNBC.

\section{Materials and methods}

Patients. A retrospective review was conducted on female patients with advanced TNBC (confirmed by pathological and immunohistochemical staining), who were treated with OXA plus S-1 at the Taixing People's Hospital (Taixing, China) between January 2011 and January 2013. The patients 
were eligible if they were confirmed to have stage IIIC or IV disease that was unsuitable for surgery or if they had developed a metastasis following surgery. Metastatic lesions were measured objectively by ultrasound, computed tomography scans or magnetic resonance imaging. Other inclusion criteria were as follows: Karnofsky Performance Status (KPS) score, $\geq 60$; expected survival, $\geq 3$ months; adequate heart, lung and renal function; white blood cell (WBC) count, $>3.5 \times 10^{9} / 1$; granulocyte (GRAN) count, $>1.5 \times 10^{9} / 1$; platelet (PLT) count, $>75 \times 10^{9} /$; liver function, $<1.5$-fold the upper limit of normal; essentially normal electrocardiograms; no peripheral neuropathy; no previous history of treatment with OXA plus S-1; no use of any chemotherapy drugs in the past month and ability to provide informed consent. The study was approved by the Institutional Review Board of the Taixing People's Hospital Ethics Committee, and all patients provided written informed consent.

Treatment, response assessments and follow-up. OXA (Jiangsu Hengrui Medicine Co., Ltd., Jiangsu, China) was administered intravenously and $135 \mathrm{mg} / \mathrm{m}^{2}$ was infused over 120 min on day 1. S-1 (Shandong New Time Pharmaceutical Co., Ltd., Shandong, China) was given orally twice daily for the first 2 weeks of a 3-week cycle; the dose of S-1 administered each time was calculated according to the patient's body surface area as follows: $<1.25 \mathrm{~m}^{2}, 40 \mathrm{mg} ; 1.25-1.5 \mathrm{~m}^{2}, 50 \mathrm{mg}$; and $>1.5 \mathrm{~m}^{2}, 60 \mathrm{mg}$. During the chemotherapy, the efficacy assessment was repeated biweekly. Cycles were repeated every 21 days for a total of 6 cycles unless disease progression was noted, in which case the regimen was modified. For patients with bone metastasis, chemotherapy and bisphosphonate treatment could be used simultaneously, while brain radiotherapy was added upon the occurrence of brain metastasis. Prior to chemotherapy, premedication consisted of a 5-hydroxytryptamine 3 receptor antagonist, and liver- and stomach-protecting treatment was administered. The adverse events of leukopenia and thrombocytopenia were treated with granulocyte colony-stimulating factor and interleukin-11, respectively. The next course of treatment was delayed when blood analysis revealed the following: WBC $<3.5 \times 10^{9} / 1$, GRAN $<1.5 \times 10^{9} / 1$ or PLT $<75 \times 10^{9} / 1$. The doses of the chemotherapy drugs were reduced by $25 \%$ in the patients who experienced grade 4 myelosuppression or grade 3 or worse peripheral neuropathy.

All patients were required to undergo complete examinations (routine blood, urine and stool; liver and renal functions; blood glucose; electrocardiography; tumor markers) prior to and following the chemotherapy. Routine blood examinations were repeated weekly and detailed records were made regarding the KPS score and weight changes during the treatment. Each patient received $\geq 2$ cycles of chemotherapy therapy, and clinical responses were confirmed based on 2 assessments performed $\geq 2$ cycles apart. The clinical responses were rated according to the Response Evaluation Criteria in Solid Tumors (10) and assigned to one of five groups: Complete response (CR), partial response (PR), marginal response, stable disease (SD) and progressive disease (PD). The overall response rate (ORR) was calculated as a ratio of the $\mathrm{CR}$ and $\mathrm{PR}$ for the entire patient population, while the disease control rate (DCR) was calculated as a ratio of the CR, PR and SD for the entire patient population. Toxicities
Table I. Patient characteristics $(\mathrm{n}=52)$.

\begin{tabular}{lr}
\hline Characteristic & $\mathrm{n}(\%)$ \\
\hline Age, years & \\
Clinical stage & $53(31-72)$ \\
IIIC & $16(30.8)$ \\
IV & $36(69.2)$ \\
Metastatic site & \\
Liver & $32(61.5)$ \\
Lymph node & $28(53.8)$ \\
Lung & $20(38.5)$ \\
Adrenal & $10(19.2)$ \\
Mediastinum & $8(15.4)$ \\
Brain & $8(15.4)$ \\
Abdominal & $8(15.4)$ \\
Bone & $6(11.5)$ \\
Others & $6(11.5)$ \\
Prior chemotherapy & \\
Taxane & $14(26.9)$ \\
Anthracycline & $10(19.2)$ \\
Anthracycline and taxane & $12(23.1)$ \\
\hline
\end{tabular}

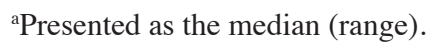

were characterized according to the National Cancer Institute Common Toxicity Criteria (11), which were divided into five grades. Progression-free survival (PFS) was calculated from the day of commencement of OXA plus S-1 administration until the day of documented progression or mortality. The OS was calculated from the start date of OXA plus S-1 to the date of mortality or last follow-up. Patients were followed up by means of letters and telephones until March 2014.

Statistical analysis. All statistical calculations were carried out using SPSS Windows version 19.9 (IBM SPSS, Armonk, NY, USA). PFS and OS were analyzed according to the Kaplan-Meier estimates and were compared in log-rank tests. $\mathrm{P}<0.05$ was considered to indicate a statistically significant difference.

\section{Results}

Patient characteristics. Table I shows the characteristics of the patients. A total of 52 patients who underwent OXA plus S-1 therapy were evaluated. The median age of the patients was 53 years (range, 31-72 years). The clinical stage of the patients was advanced to the extent that $16(30.8 \%)$ were in stage IIIC and 36 (69.2\%) were in stage IV. Liver, lymph node, lung, adrenal, mediastinum, brain, abdominal, bone and other metastases were found in 32, 28, 20, 10, 8, 8, 8, 6 and 6 patients, respectively. A total of 16 patients had a single metastatic site, and 36 patients had $\geq 2$ metastatic sites. Fourteen patients (26.9\%) had previously received taxane chemotherapy, $10(19.2 \%)$ had previously received anthracycline chemotherapy and 12 (23.1\%) had previously been treated with both anthracycline and taxane. 
Table II. Subset analysis of OS time $(n=52)$.

\begin{tabular}{|c|c|c|c|c|c|}
\hline Variables & No. of patients & Median OS (months) & $95 \% \mathrm{CI}$ & $\chi^{2}$ & P-value \\
\hline Menstruation & & & & 0.000 & 0.987 \\
\hline Pre & 28 & 13.3 & $7.1-19.5$ & & \\
\hline Post & 24 & 12.5 & $8.3-16.7$ & & \\
\hline Clinical stage & & & & 6.668 & 0.010 \\
\hline IIIC & 16 & 22.7 & 4.4-41.0 & & \\
\hline IV & 36 & 11.3 & $8.6-14.0$ & & \\
\hline Histological type & & & & 0.000 & 0.993 \\
\hline Infiltrating ductal & 40 & 13.3 & 8.9-17.7 & & \\
\hline Others & 12 & 12.3 & $2.7-21.9$ & & \\
\hline Number of organs involved & & & & 3.923 & 0.048 \\
\hline 1 & 20 & 15.7 & $5.6-25.8$ & & \\
\hline$\geq 2$ & 32 & 11.3 & $9.3-13.3$ & & \\
\hline Previous chemotherapy ${ }^{\mathrm{a}}$ & & & & 0.193 & 0.661 \\
\hline Yes & 30 & 14.5 & $5.0-24.0$ & & \\
\hline No & 22 & 12.5 & $7.4-17.6$ & & \\
\hline
\end{tabular}

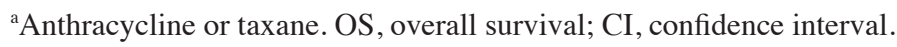

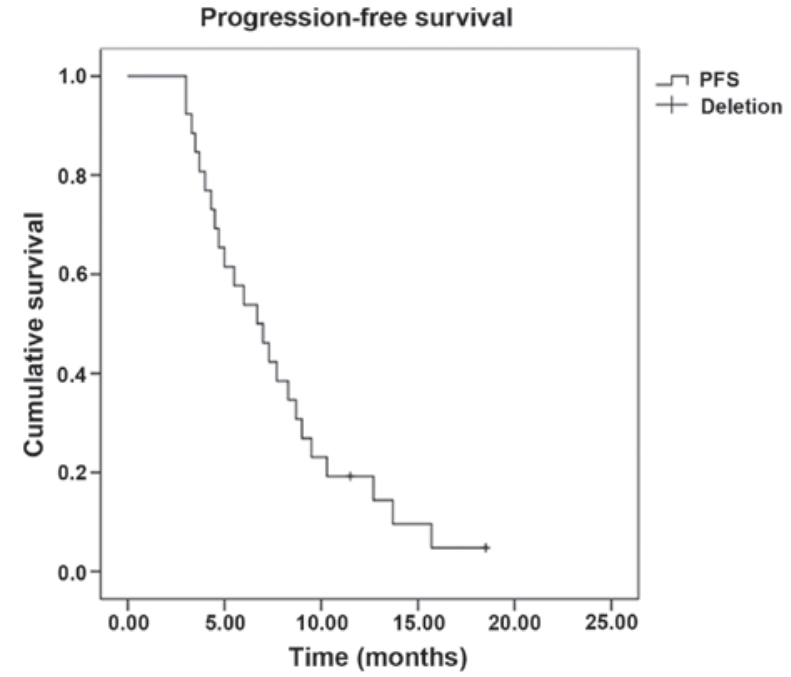

Figure 1. Kaplan-Meier curve of progression-free survival (PFS) time.

Clinical response. In total, 224 cycles of chemotherapy were performed. The median for each patient was 4 cycles, and the range was 2-6 cycles. Treatment delay occurred in 12 patients for $<7$ days due to chemotherapy toxicity. Two patients required dose reductions due to chemotherapy-induced toxicity; however, the toxicities did not lead to the complete termination of chemotherapy in any case, and there were no treatment-related mortalities. All 52 patients were evaluated for treatment efficacy. The follow-up assessments were completed by December 31, 2013, and the median follow-up time was 13.7 months (range 3.6-36.0 months). Four patients was lost to follow-up. Out of the 52 patients, 2 exhibited a CR, while PRs, SDs and PDs were noted in 16,18 and 16 patients, respectively. The ORR was $34.6 \%$ and the DCR was $69.2 \%$. The median PFS time was 6.7 months [95\% confidence interval (CI), 4.5-9.0] (Fig. 1), and the OS time was

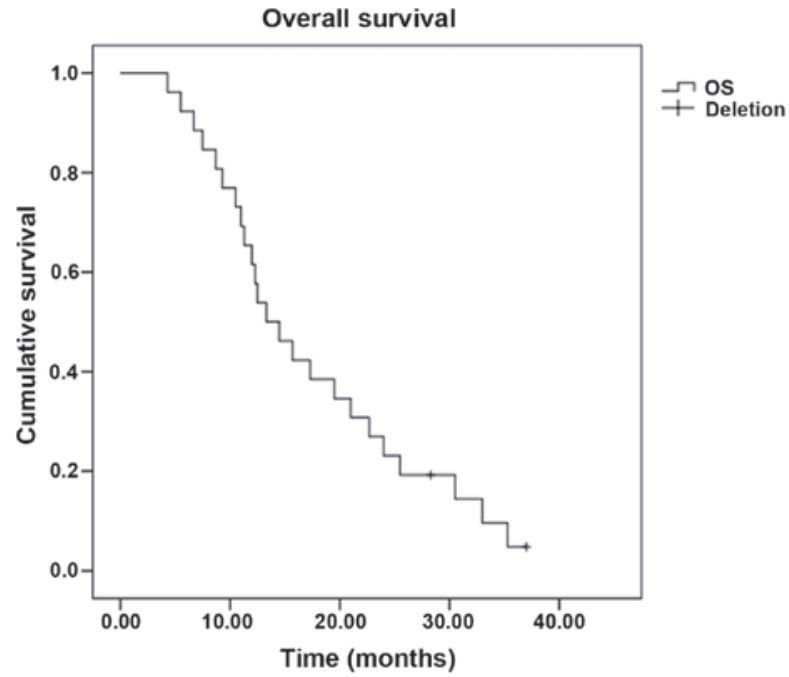

Figure 2. Kaplan-Meier curve of overall survival (OS) time.

13.3 months (95\% CI, 9.1-17.5) (Fig. 2). Subset analysis based on the clinical characteristics of the patients showed that patients who were premenopausal, had infiltrating ductal carcinoma and had previously received anthracycline or taxane chemotherapy exhibited a prolongation of survival, although the comparisons were statistically insignificant $(\mathrm{P}>0.05)$. Patients with stage IIIC disease had a statistically better median OS time than patients with stage IV disease ( 22.7 vs. 11.3 months, $\mathrm{P}=0.010)$. In addition, the median OS time of patients with a single metastatic site (15.7 months; 95\% CI, 5.6-25.8) was superior to that of patients with $\geq 2$ metastatic sites (11.3 months; 95\% CI, 9.3-13.3) $(\mathrm{P}=0.048)$ (Table II, Figs. 3 and 4).

Toxicity. The three most frequently observed adverse effects in the group were hematological toxicities, peripheral neuropathy 
Table III. Adverse events.

\begin{tabular}{lccccc}
\hline Event & Grade 1 (n) & Grade 2(n) & Grade 3 (n) & Grade 4 (n) & Grades 3+4 (\%) \\
\hline Anemia & 8 & 4 & 0 & 0 & 0.0 \\
Neutropenia & 20 & 8 & 4 & 2 & 11.5 \\
Thrombocytopenia & 4 & 2 & 0 & 0 & 0.0 \\
Nausea/vomiting & 20 & 8 & 4 & 0 & 7.7 \\
Diarrhea & 16 & 6 & 0 & 0 & 0.0 \\
Alopecia & 10 & 4 & 0 & 0 & 0.0 \\
Stomatitis & 6 & 4 & 0 & 0 & 0.0 \\
Phlebitis & 6 & 0 & 0 & 0 & 0.0 \\
Liver dysfunction & 10 & 6 & 0 & 0 & 3.8 \\
Peripheral neuropathy & 20 & 6 & 2 & 0 & 0.0 \\
Hand-foot syndrome & 12 & 6 & 0 & 0 & 0.0 \\
Pigmentation & 10 & 6 & 0 & 0 & 0.0 \\
Electrocardiogram changes & 4 & 0 & 0 & 0 &
\end{tabular}

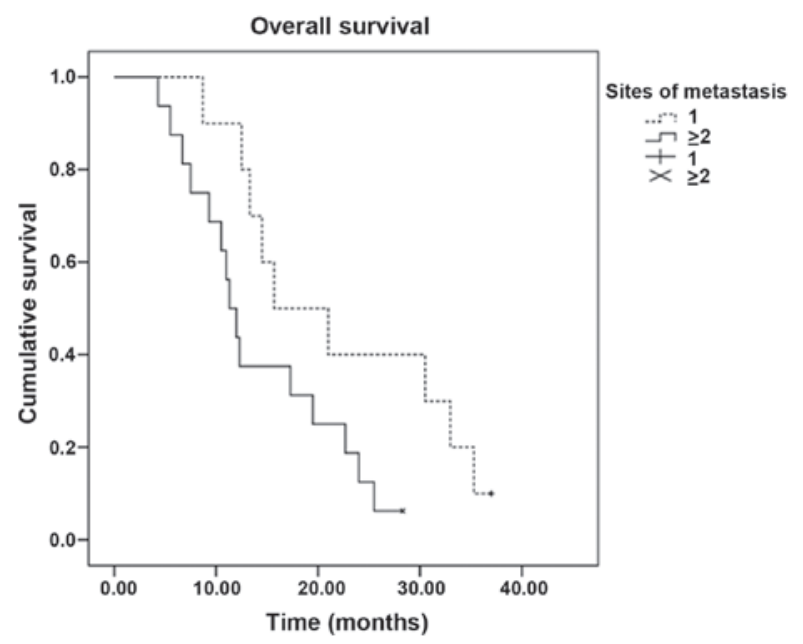

Figure 3. Kaplan-Meier curves of the overall survival time of patients with different numbers of metastatic sites. $\mathrm{x}$ and + indicate deletions.

and gastrointestinal reactions. Alopecia, liver dysfunction and hand-foot syndrome were mainly grade 1 or 2 . Grade 3 or 4 neutropenia occurred in 6 patients $(11.5 \%)$, while 4 patients $(7.7 \%)$ experienced grade 3 nausea and vomiting and 2 patients (3.8\%) experienced grade 3 peripheral neuropathy. There were no treatment-related mortalities (Table III).

\section{Discussion}

Human breast cancer can be subdivided into five molecular subtypes, distinguished by pervasive differences in their gene expression patterns: Luminal subtype A, luminal subtype B, normal breast-like, HER-2 overexpression and basal-like subtype (12). Basal-like breast cancer, by definition, is characterized by the absence of immunostaining for ER, PR and HER2, as well as the overexpression of epidermal growth factor receptor (EGFR) and cytokeratins (CK5/6, CK17 and CK14) (2). TNBC is a clinically and molecularly heterogeneous disease that encompasses more than one entity. Although TNBC and basal-like breast cancer types share numerous

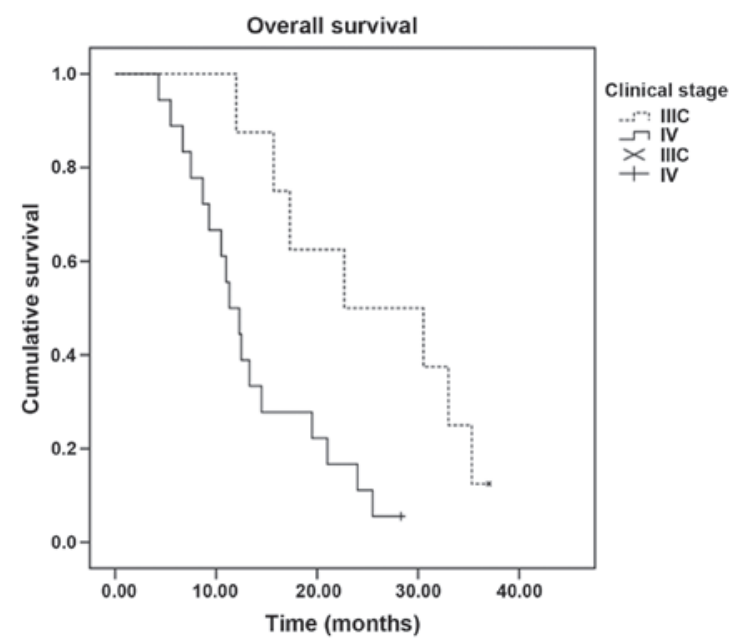

Figure 4. Kaplan-Meier curves of the overall survival time of patients with stage IIIC/IV breast cancer. $\mathrm{x}$ and + indicate deletions.

clinical and pathological characteristics, they are not identical, with basal-like tumors accounting for $\sim 75 \%$ of TNBCs (13). According to a previous study, $75 \%$ of TNBCs can be classified as BRCA1-related breast cancers, while TNBCs account for $80-90 \%$ of BRCA1-related breast cancers (14). TNBC exhibits distinct biological and clinical behavior. The majority of the tumors are high-grade, ductal carcinomas, which are associated with a larger size. Furthermore, the tumor cells exhibit an absence of androgen receptor, E-cadherin and cyclin D expression, as well as positive expression of basal cytokeratins (CK5/6, CK17), EGFR and p53 (2).

TNBC has an increased likelihood of recurrence, distant metastasis and mortality within 5 years of the diagnosis, the peak being within the first 3 years (15). Several studies have shown an increased rate of visceral metastasis, particularly spinal cord, brain, liver and lung metastasis, versus bone metastasis $(13,16,17)$. Following a diagnosis of metastatic disease, poor prognosis has also been reported (16). There are numerous predictive factors of poor prognosis, although the triple negative status itself (ER-, $\mathrm{PR}^{-}$and $\left.\mathrm{HER} 2^{-}\right)$is also 
associated with a poor prognosis $(17,18)$. p53 mutation has been reported to be associated with anthracycline- (19) and platinum- (20) based chemotherapy resistance and a poor prognosis of breast cancer $(21,22)$. The lymph node metastasis status has a significant impact on the prognosis of patients with TNBC. Hernandez-Aya et al (23) proposed that the OS rate of patients with TNBC exhibiting evidence of axillary lymph node metastasis is lower than that of patients with TNBC with no tumor metastasis. Previous studies $(24,25)$ have reported that whether the expression of ER, PR and HER-2 is concordant between metastatic axillary lymph nodes and primary lesion is an independent risk factor for TNBC.

Since patients with TNBC can benefit neither from hormone therapies nor from HER2-targeted therapies, they are more sensitive to chemotherapy than patients with other types of breast cancer. As early as 1978, a retrospective study reported that triple-negative patients had an enhanced response rate to chemotherapy as compared with non-triple-negative patients (26). Keam et al (27) analyzed 145 patients with stage II and III breast cancer who received neoadjuvant chemotherapy, and their results revealed that patients with a triple-negative phenotype showed a higher response rate (RR) than patients with a non-triple-negative phenotype ( 83.0 vs. $62.2 \%)$, as well as a higher pathologically complete RR (17.0 vs. $3.1 \%)$; both these differences were statistically significant $(\mathrm{P}<0.05)$. Deleted or mutated BRCA1 in most cases of TNBC may render them particularly susceptible to alkylating agents and platinum drugs that act by destroying the DNA (28). Currently, platinum drugs have been used in clinical trials of TNBC and have proven to be significantly sensitive among patients with TNBC. Patients who received platinum-based chemotherapy had a longer overall survival compared with patients that were administered conventional non-PBC regimens (14.5 vs. 10 months, $\mathrm{P}=0.041$ ) (29). Sirohi et al (30) retrospectively reviewed 328 breast cancer patients (62 with TNBC) treated with platinum-based chemotherapy and found that the $\mathrm{CR}$ rates were significantly higher for the patients with TNBC $(88 \%)$ than for patients with non-TNBC (51\%). In addition, the 5-year OS rates were 64 and $85 \%$, the 5-year disease-free survival rates were 57 and $72 \%$ and the ORRs were 41 and $31 \%$ for patients with TNBC and those with non-TNBC, respectively. For patients with advanced breast cancer, TNBC patients had a significantly prolonged PFS time of 6 months, compared with 4 months for patients with non-TNBC (30). From the analysis above, it can be concluded that, compared with non-TNBC, TNBC has an increased sensitivity to platinum-based chemotherapy.

OXA is a third-generation platinum chemotherapy drug that is safer and more potent than cisplatin. There are certain similarities in mechanism between OXA and cisplatin, but their chemical structures are different. OXA can bind tightly to DNA >10 times faster than cisplatin and exhibits considerably more potent cytotoxicity and broad-spectrum antitumor activity. In addition, OXA has no complete cross-resistance with cisplatin or carboplatin and is still effective in certain cases showing resistance to cisplatin or anthracycline $(7,31,32,33)$.

5-FU and OXA have a synergistic effect, and S-1 and capecitabine are 5-FU derivatives. Chemotherapy oral administration of 5-FU has become more convenient compared with intravenous administration, and does not cause phlebitis, although S-1 and capecitabine differ in terms of pharmacological and side effects $(34,35)$. A randomized, multicenter phase II trial of capecitabine versus S-1 in patients with metastatic gastric cancer demonstrated that there were no significant differences in clinical outcomes between capecitabine and S-1, with the exception of hand-foot syndrome and stomatitis, which were more frequently found in the capecitabine study arm (36). A number of clinical trials have shown that capecitabine is effective in patients with metastatic breast cancer (MBC) (37,38). S-1, a third-generation 5-FU derivative, is a combination of FT, 5-chloro-2,4-dihydropyrimidine (CDHP) and potassium oxonate (OXO). 5-FU is a derivative of FT, and is produced in liver mitochondria. CDHP acts to reversibly antagonize the activity of dihydropyrimidine dehydrogenase (DPD), which is the rate-limiting enzyme for 5-FU degradation. By inhibiting DPD, CDHP can therefore prolong the bioavailability of the 5-FU in the serum and tumors, achieving better curative effects than continuous intravenous infusion of 5-FU and preventing complications, such as phlebitis, caused by intravenous administration. The addition of OXO significantly decreases the non-hematological toxicological reactions of S-1 (39). Since its listing in Japan in 1999, numerous studies have indicated the efficacy and favorable side-effect profile of S-1 $(40,41)$. In a study investigating S-1 as a third-line or greater chemotherapy regimen in patients with MBC, one PR (3\%) and two SDs (5\%) were observed, and the median time to progression (TTP) was 84 days (42). A Japanese study showed that the ORR was $27.8 \%$, the median survival time was 19.2 months and the TTP was 6.2 months in patients with MBC who did not respond to capecitabine-based chemotherapy and then received S-1 (43). A new regimen for 16 elderly patients with advanced $\mathrm{MBC}$ revealed that chemotherapy of 2 weeks' S-1 administration followed by 1 weeks' rest was effective; the RR was $31.2 \%$, and the TTP and OS time were 5.1 and 17.9 months, respectively (44).

Previous studies have indicated that S-1 and OXA are suitable single agents for the multi-line treatment of advanced breast cancer and TNBC $(3,9,45)$. On the basis of the demonstrated efficacy, the present study retrospectively reviewed 52 patients with advanced TNBC who received S-1 combined with OXA. The results were encouraging: The ORR was $34.6 \%$, the median PFS time was 6.7 months (95\% CI, 4.5-9.0) and the median OS time was 13.3 months (95\% CI, 9.1-17.5). Subset analysis showed that patients who were premenopausal, had infiltrating ductal carcinoma and had previously been treated with anthracycline or taxane had a prolonged survival time, although statistical analysis revealed a lack of significance $(\mathrm{P}>0.05)$. The results therefore showed that prolonged survival in the present OXA- and S-1-based regimen was not significantly associated with menstrual states, pathologic types or prior chemotherapy; however, significant associations were found with clinical stage and the number of metastatic sites. Patients with stage IIIC disease had a significantly enhanced median OS time compared with patients with stage IV disease (22.7 vs. 11.3 months, $\mathrm{P}=0.010)$. The median OS time of patients with a single metastatic site (15.7 months; $95 \%$ CI, 5.6-25.8) was significantly longer than that of patients with $\geq 2$ metastatic sites (11.3 months; 95\% CI, 9.3-13.3) ( $\mathrm{P}=0.048)$. A heavy tumor load, $\geq 2$ metastatic sites and a late disease stage may therefore lead to poor prognosis. Further studies 
are required to analyze the OS times associated with different metastatic sites in a larger cohort of TNBC patients.

No unexpected adverse events occurred in this study. Both hematological and non-hematological grade 3/4 toxicities were experienced by the patients: The main hematological toxicity was neutropenia, which occurred in $11.5 \%$ of patients, while the main non-hematological toxicities were nausea and vomiting $(7.7 \%)$ and peripheral neuropathy (3.8\%). Grade $1 / 2$ toxicities, included diarrhea, liver dysfunction, stomatitis, anemia and hand-foot syndrome. The study showed that oral administration of S-1 markedly shortened the hospital stay of the patients with advanced TNBC, making it suitable for use as a palliative chemotherapeutic agent to improve the patients' quality of life.

In conclusion, S-1 plus OXA is a safe, active and well-tolerated combination and should be further investigated as a standard treatment alternative for patients with advanced TNBC. Since there are no published data on the efficacy of a combination of S-1 and OXA as a treatment for advanced TNBC, and as the present results may have some bias and limitations due to the small size of the sample, a large, randomized, prospective clinical study is warranted to confirm the results.

\section{References}

1. Vrdoljak E, Miše BP, Lukić B, et al: Long-lasting control of triple-negative metastatic breast cancer with the novel drug combination ixabepilone and capecitabine - case report. Onkologie 33: 53-56, 2010.

2. Rakha EA and Ellis IO: Triple-negative/basal-like breast cancer: Review. Pathology 41: 40-47, 2009.

3. Fei F, Chen C, Xue J, et al: Efficacy and safety of docetaxel combined with oxaliplatin as a neoadjuvant chemotherapy regimen for Chinese triple-negative local advanced breast cancer patients. A prospective, open, and unicentric Phase II clinical trial. Am J Clin Oncol 36: 545-551, 2013.

4. Oakman C, Viale G and Di Leo A: Management of triple negative breast cancer. Breast 19: 312-321, 2010.

5. Tan DS, Marchió C, Jones RL, et al: Triple negative breast cancer: Molecular profiling and prognostic impact in adjuvant anthracycline-treated patients. Breast Cancer Res Treat 111: 27-44, 2008.

6. Liu M, Mo QG, Wei CY, et al: Platinum-based chemotherapy in triple-negative breast cancer: A meta-analysis. Oncol Lett 5 : 983-991, 2013.

7. Sumpter K, Harper-Wynne C, Cunningham D, et al: Report of two protocol planned interim analyses in a randomised multicentre phase III study comparing capecitabine with fluorouracil and oxaliplatin with cisplatin in patients with advanced oesophagogastric cancer receiving ECF. Br J Cancer 92: 1976-1983, 2005.

8. Huang J, Cao Y, Wu L, et al: S-1-based therapy versus 5-FU-based therapy in advanced gastric cancer: A meta-analysis. Med Oncol 28: 1004-1011, 2011.

9. Hara F, Kiyoto S, Takahashi M, et al: Efficacy and safety of S-1 in patients with metastatic breast cancer: Retrospective review in a single institution. Oncology 79: 273-277, 2010.

10. Therasse P, Arbuck SG, Eisenhauer EA, et al: New guidelines to evaluate the response to treatment in solid tumors. European Organization for Research and Treatment of Cancer, National Cancer Institute of the United States, National Cancer Institute of Canada. J Natl Cancer Inst 92: 205-216, 2000.

11. Trotti A, Colevas AD, Setser A, et al: CTCAE v3.0: Development of a comprehensive grading system for the adverse effects of cancer treatment. Semin Radiat Oncol 13: 176-181, 2003.

12. Perou CM, Sørlie T, Eisen MB, et al: Molecular portraits of human breast tumours. Nature 406: 747-752, 2000.

13. Rastelli F, Biancanelli S, Falzetta A, et al: Triple-negative breast cancer: Current state of the art. Tumori 96: 875-888, 2010.

14. Turner N, Tutt A and Ashworth A: Hallmarks of 'BRCAness' in sporadic cancers. Nat Rev Cancer 4: 814-819, 2004.

15. Fornier $M$ and Fumoleau P: The paradox of triple negative breast cancer: Novel approaches to treatment. Breast J 18: 41-51, 2012.
16. De Laurentiis M, Cianniello D, Caputo R, et al: Treatment of triple negative breast cancer (TNBC): Current options and future perspectives. Cancer Treat Rev 36 (Suppl 3): S80-S86, 2010.

17. Rakha EA, El-Sayed ME, Green AR, et al: Prognostic markers in triple-negative breast cancer. Cancer 109: 25-32, 2007.

18. Rhee J, Han SW, Oh DY, et al: The clinicopathologic characteristics and prognostic significance of triple-negativity in node-negative breast cancer. BMC Cancer 8: 307, 2008.

19. Geisler S, Lønning PE, Aas T, et al: Influence of TP53 gene alterations and c-erbB-2 expression on the response to treatment with doxorubicin in locally advanced breast cancer. Cancer Res 61: 2505-2512, 2001.

20. Gadhikar MA, Sciuto MR, Alves MV, Pickering CR, Osman AA, Neskey DM, Zhao M, Fitzgerald AL, Myers JN and Frederick MJ: Chk1/2 inhibition overcomes the cisplatin resistance of head and neck cancer cells secondary to the loss of functional p53. Mol Cancer Ther 12: 1860-1873, 2013.

21. Dookeran KA, Dignam JJ, Ferrer K, et al: p53 as a marker of prognosis in African-American women with breast cancer. Ann Surg Oncol 17: 1398-1405, 2010.

22. Chae BJ, Bae JS, Lee A, et al: p53 as a specific prognostic factor in triple-negative breast cancer. Jpn J Clin Oncol 39: 217-224, 2009.

23. Hernandez-Aya LF, Chavez-Macgregor M, Lei X, et al: Nodal status and clinical outcomes in a large cohort of patients with triple-negative breast cancer. J Clin Oncol 29: 2628-2634, 2011.

24. Lower EE, Glass E, Blau R and Harman S: HER-2/neu expression in primary and metastatic breast cancer. Breast Cancer Res Treat 113: 301-306, 2009.

25. Lower EE, Glass EL, Bradley DA, et al: Impact of metastatic estrogen receptor and progesterone receptor status on survival. Breast Cancer Res Treat 90: 65-70, 2005.

26. Lippman ME, Allegra JC, Thompson EB, et al: The relation between estrogen receptors and response rate to cytotoxic chemotherapy in metastatic breast cancer. N Engl J Med 298: 1223-1228, 1978.

27. Keam B, Im SA, Kim HJ, et al: Prognostic impact of clinicopathologic parameters in stage II/III breast cancer treated with neoadjuvant docetaxel and doxorubicin chemotherapy: Paradoxical features of the triple negative breast cancer. BMC Cancer 7: 203, 2007.

28. Roy V, Pockaj BA, Allred JB, et al: A Phase II trial of docetaxel and carboplatin administered every 2 weeks as preoperative therapy for stage II or III breast cancer: NCCTG study N0338. Am J Clin Oncol 36: 540-544, 2013.

29. Villarreal-Garza C, Khalaf D, Bouganim N, et al: Platinum-based chemotherapy in triple-negative advanced breast cancer. Breast Cancer Res Treat 146: 567-572, 2014.

30. Sirohi B, Arnedos M,Popat S, et al: Platinum-based chemotherapy in triple-negative breast cancer. Ann Oncol 19: 1847-1852, 2008.

31. Virag P, Perde-Schrepler M, Fischer-Fodor E, et al: Superior cytotoxicity and DNA cross-link induction by oxaliplatin versus cisplatin at lower cellular uptake in colorectal cancer cell lines. Anticancer Drugs 23: 1032-1038, 2012.

32. Dunn TA, Schmoll HJ, Grünwald V, Bokemeyer C and Casper J: Comparative cytotoxicity of oxaliplatin and cisplatin in non-seminomatous germ cell lines. Invest New Drugs 15: 109-114, 1997.

33. Kang M, Jiang H, Wang S, et al: Efficacy of docetaxel combined with oxaliplatin by different administration routes in treatment of anthracycline-resistant metastatic breast cancer. Zhong Guo Zhong Liu Lin Chuang Yu Kang Fu 12: 527-529, 2005 (In Chinese).

34. Thuss-Patience PC, von Minckwitz G, Kretzschmar A, et al: Oxaliplatin and 5-fluorouracil for heavily pretreated metastatic breast cancer: A preliminary phase II study. Anticancer Drugs 14: 549-553, 2003.

35. Miura K, Kinouchi M, Ishida K, et al: 5-fu metabolism in cancer and orally-administrable 5-fu drugs. Cancers (Basel) 2: $1717-1730,2010$

36. Lee JL, Kang YK, Kang HJ, et al: A randomised multicentre phase II trial of capecitabine vs S-1 as first-line treatment in elderly patients with metastatic or recurrent unresectable gastric cancer. Br J Cancer 99: 584-590, 2008.

37. Lv H, Yan M, Zhang M, et al: Efficacy of capecitabine-based combination therapy and single-agent capecitabine maintenance therapy in patients with metastatic breast cancer. Chin J Cancer Res 26: 692-697, 2014.

38. Gelmon K, Chan A and Harbeck N: The role of capecitabine in first-line treatment for patients with metastatic breast cancer. Oncologist 11 (Suppl 1): 42-51, 2006. 
39. Shirasaka T, Tsukuda M, Inuyama Y, Taguchi T: New oral anticancer drug, TS-1 (S-1) - from bench to clinic. Gan To Kagaku Ryoho 28: 855-864, 2001 (In Japanese).

40. Koizumi W, Narahara H, Hara T, et al: S-1 plus cisplatin versus $\mathrm{S}-1$ alone for first-line treatment of advanced gastric cancer (SPIRITS trial): A phase III trial. Lancet Oncol 9: 215-221, 2008.

41. Boku N; Gastrointestinal Oncology Study Group of Japan Clinical Oncology Group: Chemotherapy for metastatic disease: Review from JCOG trials. Int J Clin Oncol 13: 196-200, 2008.

42. Shien T, Shimizu C, Akashi-Tanaka S, et al: Clinical efficacy of S-1 in pretreated metastatic breast cancer patients. Jpn J Clin Oncol 38: 172-175, 2008.
43. Yamamoto D, Iwase S, Yoshida H, et al: Efficacy of S-1 in patients with capecitabine-resistant breast cancer-Japan Breast Cancer Research Network (JBCRN) 04-1 trial. Anticancer Res 30: 3827-3831, 2010.

44. Fujii K, Kosaka J, Mouri Y, et al: Chemotherapy of a 2-week S-1 administration followed by 1-week rest for advanced and metastatic breast cancer. Gan To Kagaku Ryoho 38: 1467-1470, 2011 (In Japanese).

45. Saeki T: Clinical benefit of S-1 in metastatic breast cancer. Gan To Kagaku Ryoho 33 (Suppl 1): 202-206, 2006 (In Japanese). 